\title{
ON SYMPLECTIC COBORDISM OF REAL PROJECTIVE PLANE
}

\author{
M. BAKURADZE
}

Abstract

This note answers a question of V. V. Vershinin concerning the properties of Buchstaber's elements $\theta_{2 i+1}(2)$ in the symplectic cobordism ring of the real projective plane. It is motivated by Roush's famous result that the restriction of these elements to the projective line is trivial, and by the relationship with obstructions to multiplication in symplectic cobordism with singularities.

The symplectic cobordism of real projective space is of genuite interest to algebraic topologists, since it is expected to form an essential ingredient of any extension of the theory of formal group lows to the quaternionic case. Buctstaber's elements arise from the quaternionic analogue (1) of Quillen's well known formula about the stable Euler class for the universal double covering. These elements were introduced by V. M. Buchstaber in $[\mathbf{2}]$ and developed in his unpublished work. In the same work Buchstaber gives another proof of Roush's result by a different method and establishes the certain functional dependence algorithm between his elements and Roush's elements $\gamma_{j}$ in $M S p^{*}\left(R P^{\infty}\right)$. He also gives the formula (1) and calculations of Ladweber-Novikov operations on Roush's elements.

In [3] two properties of the elements $\theta_{i}$ in $M S p^{*}\left(R P^{2}\right)$ are given (see below). In this paper we give some other properties of these elements. For the proof of our result we use the reasoning similar to that of Buchstaber's work and $[\mathbf{3}]$.

Let $\xi \rightarrow R P^{n}$ be the canonical real line bundle and $\zeta \rightarrow H P^{\infty}$ be the canonical symplectic line bundle. Since there is an additive isomorphism

$$
M S p^{*}\left(R P^{\infty} \wedge H P^{\infty}\right) \simeq M S p^{*}\left(R P^{\infty}\right)[x],
$$

1991 Mathematics Subject Classification. 55N22, 55R12.

Key words. Transfer, Euler class, projective space, symplectic cobordism.

Supported by a grant from the Georgian Academy of Sciences and INS grant RVJ 200. 
where $x=e(\zeta)$ is the Euler class of $\zeta$, one sees that the Euler class of the symplectic virtual bundle $\left((\xi-1) \otimes_{R}(\zeta-4)\right)$ has the form

$$
e\left((\xi-1) \otimes_{R}(\zeta-4)\right)=\sum_{i \geq 1} a_{i} x^{i},
$$

for some elements $a_{i} \in M S p^{4-4 i}\left(R P^{\infty}\right)$.

For the restrictions of $a_{i}$ to the symplectic cobordism ring of the $n$-dimensional real projective space $R P^{n}$ the notation $\theta_{i}(n)$ is used. These elements $a_{i}$ and $\theta_{i}(n)$ have been studied by $\mathrm{V}$. Buchstaber in $[2]$.

Since $R P(1)=S^{1}$ and $\widetilde{M S p^{1}}\left(S^{1}\right) \simeq Z$, the restriction of $\theta_{i}(n)$ on $R P(1)$ has the form $\theta_{i}(1)=s_{1} \theta_{i}$ for the generator $s_{1} \in \widetilde{M S p^{1}}\left(S^{1}\right)=Z$ and for some coefficients $\theta_{i} \in M S p^{3-4 i}(p t)$. These elements $\theta_{i}$ are called Ray elements. The elements $\theta_{1}$ and $\theta_{2 i}, i \geq 1$ are indecomposable and have order $2[\mathbf{4}]$ and $\theta_{2 i+1}=0[\mathbf{5}]$.

Let $r$ be the generator of $M S p^{2}\left(R P^{2}\right)=Z_{2}$. Recall two formulae in $M S p^{*}\left(R P^{2}\right)$ from [3].

$$
2 \theta_{i}(2)=\theta_{1} \theta_{i} r
$$

and

$$
\theta_{i}(2) \theta_{j}(2)=\theta_{i} \theta_{j} r
$$

Let us write $\phi_{0}=\theta_{1}$ and $\phi_{i}=\theta_{2 i}, i \geq 1$.

Theorem. In $M S p^{*}\left(R P^{2}\right)$ the following relations hold

$$
\begin{aligned}
& \theta_{8 n+7}(2)=0 \\
& \theta_{8 n+3}(2)=\phi_{2 n+1}^{2} r \\
& \theta_{4 k+1}(2)=\sum_{i=0}^{k-1} \phi_{2 i+1} \phi_{2(k-i)} r
\end{aligned}
$$

for $n \geq 0$ and $k \geq 1$.

Proof: We use Becker-Gottlieb transfer method [1]. Namely we need two formulas: First as proved by Buchstaber $[\mathbf{3}]$ for the transfer map $\tau(\pi)$ of the double covering $\pi: S^{\infty} \rightarrow R P^{\infty}$ there is the formula

$$
\tau^{*}(\pi)(1)=2+\sum_{k=1}^{\infty} a_{k} y^{k-1},
$$

where $y=e(\xi)$. So for the case $\pi: S^{2} \rightarrow R P^{2}$ we obtain

$$
\tau(\pi)^{*}(1)=2+\theta_{1}(2) .
$$

The second formula which we need is the following [5]. 
Let $p$ be a double covering $p: X \rightarrow B$, let $\eta$ be the symplectic line bundle $\eta \rightarrow X, \eta_{\text {! }}$ be the Atiyah transfer of $\eta, \tau(p)$ be the transfer map for $p$ and $f: B \rightarrow R P^{\infty}$ be the classifying map of the real line bundle associated with the double covering $p$. Then for some elements $\gamma_{i}$ from $M S p^{4-8 i}\left(R P^{\infty}\right)$ the following formula holds

$$
\tau(p)^{*}\left(P_{1}(\eta)\right)=P_{1}\left(\eta_{!}\right)+\sum_{i \geq 0} f^{*}\left(\gamma_{i}\right) P_{2}^{i}\left(\eta_{!}\right)
$$

in $M S p^{4}(B)$, where $P_{1}, P_{2}$ denote symplectic Pontrjagin classes. Applying (3) to the transfer map $\tau=\tau(\pi \times 1)$ for the double covering

$$
\pi \times 1: S^{2} \times H P^{\infty} \rightarrow R P^{2} \times H P^{\infty}
$$

and taking into account

$$
\begin{gathered}
\zeta !=\zeta+\xi \otimes_{R} \zeta \\
P_{1}(\zeta !)=x+x+\sum_{i \geq 1} \theta_{i}(2) x^{i} ;
\end{gathered}
$$

and

$$
P_{2}\left(\zeta_{!}\right)=x\left(x+\sum_{i \geq 1} \theta_{i}(2) x^{i}\right)
$$

we have

$$
\tau^{*}(x)=x+x+\sum_{i \geq 1} \theta_{i}(2) x^{i}+\sum_{j \geq 1} f^{*}\left(\gamma_{j}\right)\left(1+\sum_{i \geq 1} \theta_{i}(2) x^{i-1}\right)^{j} x^{2 j} .
$$

On the other hand by (2) we have

$$
\tau^{*}(x)=\left(2+\theta_{1}(2)\right) x
$$

and we obtain

$$
\sum_{i \geq 2} \theta_{i}(2) x^{i}=-\sum_{j \geq 1} f^{*}\left(\gamma_{j}\right)\left(1+\sum_{i \geq 1} \theta_{i}(2) x^{i-1}\right)^{j} x^{2 j} .
$$

The diagonal of $R P^{2} \wedge R P^{2}$ coincides with $R P^{1} \wedge R P^{1}$ i. e. with $S^{2}$, and the diagonal map $R P^{2} \rightarrow R P^{2} \wedge R P^{2}$ factors as composition of the projection $R P^{2} \rightarrow S^{2}$ onto the top cell with the inclusion of the bottom cell. Then the triple diagonal map $R P^{2} \rightarrow R P^{2} \wedge R P^{2} \wedge R P^{2}$ is nullhomotopic. This means that for any $\alpha, \beta \in M S p^{*}\left(R P^{2}\right)$ we have $\alpha \beta=\alpha_{1} \beta_{1} r$, where $s_{1} \alpha_{1}$ and $s_{1} \beta_{1}$ are restrictions of $\alpha$ and $\beta$ to $R P^{1}=$ $S^{1}$. In particular, all triple products in $M S p^{*}\left(R P^{2}\right)$ are zero.

After the remarks on double and triple products the proof is completed by (4) and induction on $i$. 


\section{References}

[1] J. C. Becker and D. H. Gottlieb, The transfer map and fibre bundles, Topology 14 (1975), 1-12.

[2] V. M. Buchstaber, Characteristic classes in cobordisms and topological application of theories of one and two-valued formal groups, Itogi Nauki i Tekhniki 10 (1978), 5-178.

[3] V. Gorbunov and N. Ray, Orientations of Spin bundles and symplectic cobordism, Publ. Res. Inst. Math. Sci. 28(1) (1992), 39-55.

[4] N. RAY, Indecomposables in Tors $\mathrm{MSp}_{*}$, Topology 10 (1971), 261-270.

[5] F. W. Roush, On some torsion classes in symplectic bordism, unpublished.

Institute of Mathematics

Department of Topology and Geometry

380093 Tbilisi

Georgia

E-mail address: maxo@rmi.acnet.ge

Primera versió rebuda el 19 de juliol de 1999, darrera versió rebuda el 7 de febrer de 2000 . 